\title{
GeneXpert MTB/RIF Assay: A Revolutionizing Method for Rapid Molecular Detection of Mycobacterium Tuberculosis in Comparison to Other Conventional Methods
}

\author{
Ehab Badran Rakha ${ }^{1 *}$ and Mohammed Abdel Razek Abdel Hakeem² \\ ${ }^{1}$ King Khalid Hospital, Hail, KSA, Clinical Pathology Department, Mansoura faculty of \\ Medicine, Mansoura University, Egypt \\ ${ }^{2}$ Clinical Pathology Department, Minia University Hospitals, Minia, Egypt \\ *Corresponding author
}

\begin{tabular}{|l|}
\hline K e y w or d s \\
Tuberculosis, \\
GeneXpert \\
MTB/RIF, \\
MGIT960 culture, \\
Ziehl-Neelsen stain. \\
\hline Article Info \\
\hline Accepted: \\
23 September 2017 \\
Available Online: \\
10 October 2017 \\
\hline
\end{tabular}

\section{Introduction}

Mycobacterium tuberculosis (MTB) is the causative agent of Tuberculosis (TB) which remains the leading cause of morbidity and mortality worldwide. It is airborne disease that primary infects the lungs (pulmonary TB) (Hosne et al., 2016). culture and $\mathrm{ZN}$ stain.

\section{A B S T R A C T}

Tuberculosis (TB) remains the leading cause of morbidity and mortality worldwide that is caused by Mycobacterium tuberculosis (MTB). Conventional cultures are highly sensitive but time-consuming, whereas Ziehl-Neelsen $(\mathrm{ZN})$ stain is a rapid method with high specificity but has lower sensitivity. The GeneXpert MTB/RIF assay represents a great breakthrough in rapid diagnosis of TB which is not only important in treatment but also has a vital role to prevent its transmission in the community. The aim of this study is to throw a light on the importance of GeneXpert assay as a rapid and reliable test for the diagnosis of TB compared to conventional MGIT960 culture and ZN microscopy. A total of 380 sputum samples and 55 extrapulmonary samples (37 pleural fluids, 11 CSF, 3 peritoneal fluids, 2 pus and 2 synovial fluids) were taken from 435 patients clinically suspected to have TB. GeneXpert, MGIT960 liquid culture and ZN stain were done for all samples. Out of 435 cases, 41 cases $(9.4 \%)$ were positive for TB by bacteriological, clinical, radiological and/or pathological evidence. From 41 positive TB cases 40,35 , and 30 cases were positive by GeneXpert assay, MGIT960 and ZN stain respectively. The overall sensitivity, specificity, negative predictive value (NPV) and positive predictive value (PPV) of GeneXpert were 97.6\%, $100 \%, 99.7 \%$ and $100 \%$ respectively. On the other hand, the sensitivity, specificity, NPV and PPV of MGIT960 culture were $85.4 \%$, $99.4 \%, 98.5 \%$ and $94.6 \%$, while for $\mathrm{ZN}$ stain were $73.2 \%, 99.4 \%, 99.5 \%$ and $93.8 \%$ respectively. GeneXpert is a simple, rapid, highly sensitive (97.6\%) and specific (100\%) method that greatly speeds up the detection of MTB compared to conventional MGIT960 
Nigeria, Pakistan and South Africa (WHO, 2016).

The GeneXpert MTB/RIF assay (Cepheid, Inc., Sunnyvale, CA, USA) represents a great breakthrough in the rapid diagnosis of TB which is essential for both early disease treatment and infection control. It detects both Mycobacterium tuberculosis complex (MTBC) and rifampicin (RIF) resistance by PCR amplification of the 81-bp fragment of the MTB rpoB gene in 2 hours (Arzu et al., 2011). This gene is known to be specific for TB diagnosis and its mutation is associated with resistance to RIF. The assay not only provides the advantage of rapid diagnosis within 2 hours but also detects even low numbers of MTB in various specimens (Maynard-Smith et al., 2014).

Conventional cultures are highly sensitive but time-consuming, whereas $\mathrm{ZN}$ stain is a rapid method with high specificity but has lower sensitivity (Nakwon et al., 2013).

\section{Aim of the work}

The aim of this study is to throw a light on the importance of GeneXpert assay as a rapid and reliable test for the diagnosis of TB compared to conventional MGIT960 culture and ZN microscopy.

\section{Materials and Methods}

This study was conducted in Microbiology Laboratory, King Khalid Hospital, Hail, KSA between January 2016 and February 2017. A total of 380 sputum samples and 55 extrapulmonary samples ( 37 pleural fluids, 11 CSF, 3 peritoneal fluids, 2 pus and 2 synovial fluids) were taken from 435 patients clinically suspected to have TB.

Nonsterile clinical specimens were processed by the conventional N-acetyl-L-cysteine-
$\mathrm{NaOH}$ method for digestion, decontamination and concentration (Shafiq et al., 2016).

Processed samples were divided into three parts; one part was immediately tested using GeneXpert, second part used for ZN smear microscopy and the third part for MGIT960 liquid culture.

\section{AFB smears}

All the smears were stained by the $\mathrm{ZN}$ method and examined microscopically (Lombardi et al., 2017).

MGIT960 [Becton Dickinson, Sparks, MD, USA]

MGIT960 is a non-radiometric automated isolation system for recovery of MTB. The MGIT tube contains $7 \mathrm{ml}$ of $7 \mathrm{H} 9$ medium and supplemented with $0.8 \mathrm{ml}$ of Oleic AcidAlbumin-Dextrose-Catalase [OADC] along with PANTA [Polymyxin B-Amphotericin BNalidixic acid -Trimethoprim - Azlocillin] (Siddiqi et al., 2006). To this tube, $0.5 \mathrm{ml}$ of decontaminated sample was added. If there was no growth of MTB after 6 weeks, the culture was considered negative. When the tubes were flagged positive, $\mathrm{ZN}$ staining and culture on 5\% sheep blood agar were performed from the tube directly to rule out any contamination as per the manufacturer's instructions (Hosne et al., 2016).

\section{GeneXpert assay method}

GeneXpert MTB/RIF assay can detect MTBC and associated RIF resistance directly from clinical samples using ultrasensitive nested real time PCR (Vadwai et al., 2011).The assay integrates sample processing and PCR in a single-use plastic cartridge containing all reagents required for bacterial lysis, nucleic acid extraction, amplification and amplicon detection (Evans, 2011). 
GeneXpert testing was performed according to the manufacturer's instructions. Decontaminated samples were added to sample reagent with 1:3 dilutions, manually mixed two times and kept for $15 \mathrm{~min}$ at room temperature. Two $\mathrm{ml}$ of the processed material was transferred to the test cartridge and loaded into the test platform of the machine (Tang et al., 2017).

Mycobacterium tuberculosis complex is detected by five overlapping probes that collectively are complementary to the entire $81 \mathrm{bp}$ rpoB core region of RIF-susceptible MTB (wild type) and are labeled with a differently colored fluorophore (Lawn and Nicol, 2011). Positive results were reported when at least two of the five probes give positive signals with a cycle threshold (CT) of $\leq 38$ cycles. Also, a semi-quantitative estimate of positive samples depending on CT range was given (high, <16; medium, 16-22; low, 22-28; very low, >28). Each test includes a Sample Processing Control (SPC) and Probe Check Control (PCC). The SPC monitor adequate processing of the samples and the presence of inhibitors in the PCR reaction. The PCC verifies reagent rehydration, PCR tube filling in the cartridge, probe integrity and dye stability (Blakemore et al., 2011).

\section{Results and Discussion}

Out of 435 cases, 41 cases $(9.4 \%)$ were positive for TB by bacteriological, clinical, radiological and/or pathological evidence. The upper hand for MTB detection was demonstrated by GeneXpert assay (40/41; 39 sputum and on pus samples), followed by MGIT960 (35/41) and ZN stain (30/41).Only one sample was reported negative by the GeneXpert assay while itsMGIT960 culture result was positive, in addition to the presence of clinical and radiological evidence of TB. Also, there were 6 and 11 negative samples by MGIT culture and ZN stain respectively, but they were positive by the GeneXpert. All these data are shown in table 1 and figure 1.

In table 2, all high and medium positive results by GeneXpert MTB/RIF assay were positive by both MGIT960 culture and ZN stain, whereas all very low positive cases by GeneXpert MTB/RIF assay were negative by MGIT960 culture and ZN stain. Also, all low positive cases by GeneXpert MTB/RIF assay were positive by MGIT960 culture and negative by ZN stain.

Regarding table 3 , among the 435 specimens, 30 samples were positive by all three methods, 4 by GeneXpert and MGIT, 6 by GeneXpert only and one by MGIT only.

Of 395 negative cases by GeneXpert assay, 3 and 2 cases were positive by MGIT and ZN stain respectively. The two positive cases by MGIT culture and ZN stain were identified as Mycobacterium Other Than Tuberculosis (MOTT). The third positive one by MGIT and negative by ZN stain was MTB by clinical, radiological and pathological evidence.

In order to start appropriate treatment, rapid diagnosis of TB is very important. Turnaround time of GeneXpert assay to detect MTBC is only 2 hours compared to $15 \pm 5$ days and 2 hours for MGIT culture and ZN stain respectively (Table 4).

By using a combination of bacterial, clinical, radiological and/or histopathological evidence of TB as the reference standard, the sensitivity, specificity, negative and positive predictive values for GeneXpert assay were 97.6, $100 \%, 99.7 \%$ and $100 \%$ respectively. Also, the sensitivity, specificity, negative and positive predictive values of MGIT culture were $85.4 \%, 99.4 \%, 98.5 \%$ and $94.6 \%$ and for ZN stain were $73.2 \%, 99.4 \%, 99.5 \%$ and $93.8 \%$ respectively (Table 5). The rapid detection of MTB is essential for both early disease management and infection control. 
The GeneXpert MTB/RIF assay is a cartridge-based, automated and rapid molecular assay that performs rapid TB diagnosis within 2 hours. In this study, our aim is to throw a light on the importance of GeneXpert MTB/RIF assay as a rapid and reliable test for the diagnosis of TB compared to conventional methods.

Out of 435 cases included in our study, 41 cases $(9.4 \%)$ were positive for TB by bacteriological, clinical, radiological and/or pathological evidence. Higher percentage of TB around $25 \%$ was demonstrated by Nakwon et al., (2013), Arzu et al., (2011) and Agrawal et al., (2016). In India, Raj et al., (2012) and Sajed et al., (2014), found that positive cases represent $51.7 \%$ (out of 547 suspected patient) and 37\% (out of 100 suspected patients) respectively.

This high percentage of TB in these studies compared to our study could be explained by the fact that South Korea and Turkey were considered as countries with intermediate burden of TB (Kim et al., 2012), while the world's largest burden of TB was found in India (Agrawal et al., 2016).

The highest detection of MTB was demonstrated by GeneXpert assay (40/41; 39 sputum and on pus samples), followed by MGIT960 (35/41) and ZN stain (30/41). Similar results were demonstrated by Agrawal et al., 2016, Bajrami et al., 2016) and Tang et al., 2017.

Table.1 Number of positive cases by different methods in relation to all studied cases and positive TB cases

\begin{tabular}{|l|l|l|l|l|}
\hline \multirow{2}{*}{ Methods } & \multicolumn{2}{|l|}{$\begin{array}{l}\text { Total number of studied } \\
\text { cases }(\mathrm{n}=435)\end{array}$} & $\begin{array}{l}\text { Total number of positive cases } \\
(\mathrm{n}=41)\end{array}$ \\
\cline { 2 - 5 } & Positive & negative & Positive & Negative \\
\hline GeneXpert assay & $40(9.2 \%)$ & 395 & 40 & 1 \\
\hline MGIT 960culture & $37(8.5 \%)$ & 398 & 35 & 6 \\
\hline ZN stain & $32(7.4 \%)$ & 403 & 30 & 11 \\
\hline
\end{tabular}

Table.2 Relation between semi-quantitative GeneXpert assay results and conventional methods (MGIT960 culture and ZN stain).

\begin{tabular}{|l|l|l|}
\hline $\begin{array}{l}\text { Semi-quantitative GeneXpert } \\
\text { assay }(\mathrm{n}=40)\end{array}$ & Positive MGIT culture & Positive ZN stain \\
\hline High positive(n=21) & 21 & 21 \\
\hline Medium positive(n=9) & 9 & 9 \\
\hline Low positive(n=4) & 4 & 0 \\
\hline Very low positive(n=6) & 0 & 0 \\
\hline Total & 34 & 30 \\
\hline
\end{tabular}


Table.3 Comparison of GeneXpert assay with MGIT culture and ZN stain

\begin{tabular}{|l|l|l|l|l|}
\hline \multirow{2}{*}{} & \multicolumn{3}{|l|}{ MGIT culture } & \multicolumn{2}{l|}{ ZN stain } \\
\cline { 2 - 5 } & positive & negative & positive & Negative \\
\hline GeneXpert assay & & & & \\
\hline Positive $(\mathrm{n}=40)$ & 34 & 6 & 30 & 10 \\
\hline Negative $(\mathrm{n}=395)$ & 3 & 392 & 2 & 393 \\
\hline Total & 37 & 398 & 32 & 403 \\
\hline
\end{tabular}

Table.4 Turnaround time (TAT) of TB detection by different methods

\begin{tabular}{|l|l|l|}
\hline Methods & TAT of positive results & TAT of negative results \\
\hline GeneXpert assay & 2 hours & 2 hours \\
\hline MGIT culture & $15 \pm 5$ & 6 weeks \\
\hline ZN stain & 2 hours & 2 hours \\
\hline
\end{tabular}

Table.5 Sensitivity, specificity and predictive values of different methods in the detection of positive TB cases ( 41 cases)

\begin{tabular}{|l|l|l|l|l|}
\hline Methods & Sensitivity & Specificity & NPV & PPV \\
\hline $\begin{array}{l}\text { GeneXpert } \\
\text { assay }\end{array}$ & $97.6 \%(40 / 41)$ & $100 \%(394 / 394)$ & $99.7 \%(394 / 395)$ & $100 \%(40 / 40)$ \\
\hline $\begin{array}{l}\text { MGIT } \\
\text { culture }\end{array}$ & $860.4 \%(35 / 41)$ & $99.4 \%(394 / 396)$ & $98.5 \%(394 / 400)$ & $94.6 \%(35 / 37)$ \\
\hline ZN stain & $73.2 \%(30 / 41)$ & $99.4 \%(394 / 396)$ & $99.5 \%(394 / 405)$ & $93.8 \%(30 / 32)$ \\
\hline
\end{tabular}

Fig.1 Number of positive cases by different methods in relation to all positive TB cases ( $\mathrm{n}=41$ )

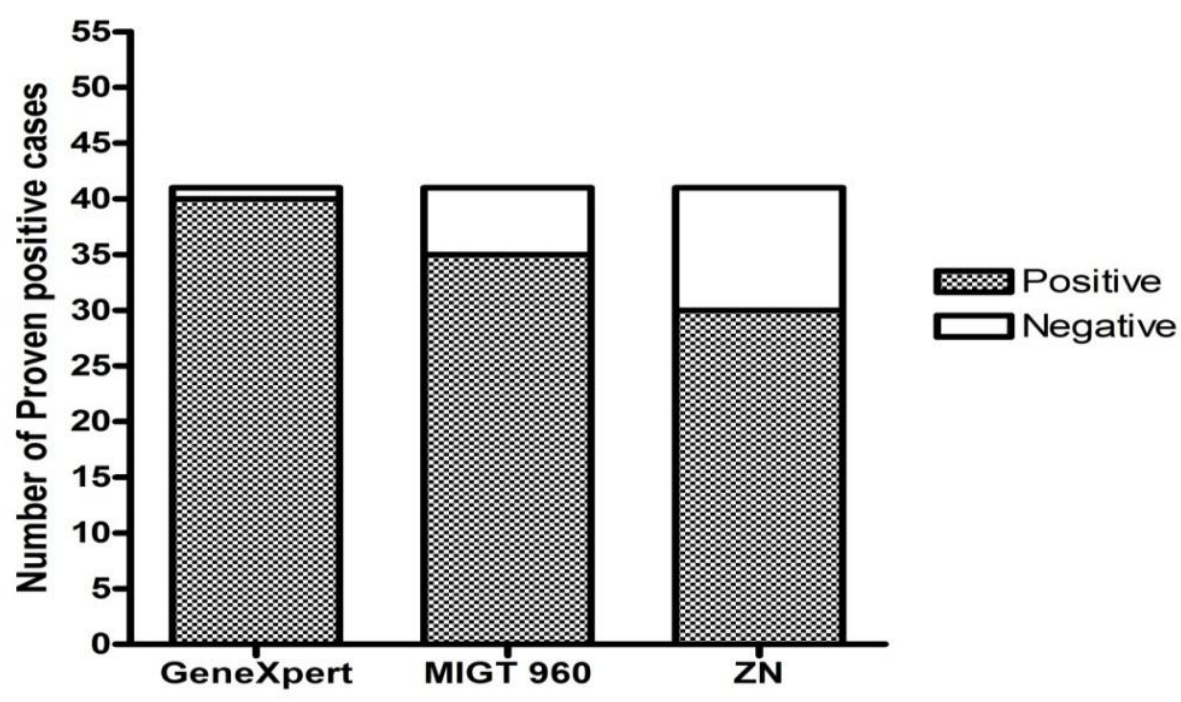


No false positive results were reported by GeneXpert, but there were 2 cases by MGIT culture and $\mathrm{ZN}$ stain. These two false positive cases (positive by both MGIT and ZN and negative by GeneXpert) were identified as MOTT. This is because GeneXpert detects only certain part of DNA which is specific to MTBC, but MGIT culture and ZN stain cannot differentiate between MTBC and MOTT. On the contrary, none of MGIT culture and ZN positive samples for MTB was negative by GeneXpert except only one sample that was positive by MGIT and negative by GeneXpert, whereas, there were 6 and 11 false negative samples (positive by GeneXpert) by MGIT culture and ZN stain respectively.

This discordance between GeneXpert and MGIT culture was also reported by Agrawal et al., 2016, Bajrami et al.,2016and tang et al.,2017who found that 9,6and 19 samples were GeneXpert positive and culture negative respectively. Also, the same previous authors found that 2,2 and 13 samples were negative by GeneXpert and positive by culture respectively. This false negative result by GeneXpert could be due to the presence of few nucleic acid material or PCR inhibitors in the specimens, meanwhile, false negative results (positive by GeneXpert and negative by culture) could be due to the very low detection range of GeneXpertor death of some bacilli during decontamination (PCR test amplifies DNA of live or dead bacilli, but MGIT culture only detect living MTB).

In our study, by using a combination of bacterial, clinical, radiological and/or histopathological evidence of $\mathrm{TB}$ as the reference standard, overall sensitivity, specificity, NPV and PPV of GeneXpert were97.6, $100 \%, \quad 99.7 \%$ and $100 \%$ respectively. Also, the sensitivity, specificity, negative and positive predictive values of MGIT culture were $85.4 \%, 99.4 \%, 98.5 \%$ and $94.6 \%$ and for $\mathrm{ZN}$ stain were $73.2 \%, 99.4 \%$,
99.5\% and $93.8 \%$ respectively (Table 5). This higher sensitivity and specificity of GeneXpert compared to MGIT culture and $\mathrm{ZN}$ stain is due to the ability of GeneXpert to detect very low level of MTB (Table 2).

In conjunction with our study, Pandey et al., 2017, found that the overall sensitivity, specificity, PPV and NPV of GeneXpert MTB/RIF assay were to be $98.6 \%, 100 \%$, $100 \%$ and $93.8 \%$ respectively. Furthermore, Singh et al., 2016, demonstrated a sensitivity of $100 \%$ and specificity of $100 \%$ for pulmonary samples by using GeneXpert assay. In addition, Agrawal et al., 2016, reported that the sensitivity and specificity of GeneXpert in sputum were $100 \%$ and $90 \%$ that is compatible with the study of Sharma et $a l ., 2015$ who found a sensitivity of $96.9 \%$ and a specificity of $99.8 \%$.

On the other side, Moussa et al., 2016, found that the sensitivity and specificity of the GeneXpert assay were $93 \%$ and $98.3 \%$ respectively. This relatively low sensitivity compared to our results could be due to a low number of patients (218) and different study design. Similarly, according to the study done by Arzu et al., (2011), 110 tuberculosis patients (62 pulmonary TB and 48 extra pulmonary TB) were diagnosed. The sensitivity, specificity, NPV and PPV of the GeneXpert were $70 \%, 100 \%, 90.6 \%$, and $100 \%$ respectively. This difference in sensitivity between this study and our study could be explained by the presence of large numbers of paucibacillary extra pulmonary TB cases (48 out of 110) with a very low numbers of organisms which were under the limits of detection of the GeneXpert. The detection limit of GeneXpert is about 4.5 genomes per reaction or $131 \mathrm{cfu} / \mathrm{ml}$ (Helb et al., 2010).

The time factor is very important in the diagnosis of TB to start appropriate treatment. In the present study, turnaround time of 
GeneXpert assay is only 2 hours compared to $15 \pm 5$ days for MGIT960 culture. Although the turnaround time of $\mathrm{ZN}$ smear was as rapid as GeneXpert, the sensitivity of the GeneXpert was much higher.

In a similar study by Sajed et al., (2014) and Tang et al., (2017), the mean detection time of GeneXpert assay was only 2 hours compared to $3.5 \pm 0.8$ hours for $\mathrm{Zn}$ smear, $12 \pm 5$ days for positive MGIT Culture and 42 days for a negative result of culture.

GeneXpert is a simple, rapid, highly sensitive (97.6\%) and specific (100\%) method that greatly speeds up the detection of MTB compared to conventional MGIT 960 culture and $\mathrm{ZN}$ stain.

\section{Acknowledgements}

The authors acknowledge the great effort of Khalid Abdelaziz Al Nazhain carrying out the practical part. We are also thankful to Waleed Mansi Al shammari, director of laboratory department for his great support.

\section{References}

Agrawal, M., Bajaj, A., Bhatia, V., Dutt, S. 2016. Comparative study of GeneXpert with $\mathrm{ZN}$ stain and culture in samples of suspected pulmonary tuberculosis. Journal of Clinical and Diagnostic Research, 10(5): DC09-DC12.

Arzu, N.Z., Sezai, T. and Cengiz, C.2011. Evaluation of the GeneXpert MTB/RIF assay for rapid diagnosis of tuberculosis and detection of rifampin resistance in pulmonary and extrapulmonary specimens. J Clin. Microbiol, Dec., p. 4138-4141.

Bajrami, R., Mulliqi, G., Kurti, A., Lila, G., Raka, L. 2016. Comparison of GeneXpert MTB/RIF and conventional methods for the diagnosis of tuberculosis in Kosovo. J. Infect. Dev. Ctries, 10(4):418-422.

Blakemore, R., Story, E., Helb, D., Kop, J.,
Banada, P., Owens M.R., Chakravorty, S., Jones, M. and Alland, D. 2010. Evaluation of the analytical performance of the Xpert MTB/RIF assay. J Clin Microbiol, 48(7): 2495-501.

Evans, C.A., 2011. GeneXpert-a game-changer for tuberculosis control? PLOS Med, 8(7).

Helb, D., Jones, M., Story, E., Boehme, C., Wallace, E., Ho, K. 2010. Rapid detection of Mycobacterium tuberculosis and rifampin resistance by use of on-demand, near-patient technology. Journal Clin. Microbiol, 48: 229-237. doi: 10.1128/JCM.01463-09. pmid:19864480.

Hosne, J., Sanya, T.J., Zakir, H.H., Yusuf, M.A., Imtiaz, A., Aleya, F. and Rafia, P. 2016. Diagnostic evaluation of GeneXpert MTB/RIF assay for the detection of rifampicin resistant Mycobacterium tuberculosis among pulmonary tuberculosis patients in Bangladesh. Journal of Tuberculosis Research, 4: 55-60.

Kim, S.Y., Kim, H., Kim, S.Y., Ra, E.K., Joo, S.I., Shin, S., Seong, M.W., Yoo, C.G., Kim, E.C., and Park, S. 2012. The Xpert ${ }^{\circledR}$ MTB/RIF assay evaluation in South Korea, a country with an intermediate tuberculosis burden. The International Journal of Tuberculosis and Lung Disease, 16 (11):1471-1476.

Lawn, S.D., and Nicol, M.P. 2011. Xpert® MTB/RIF assay: development, evaluation and implementation of a new rapid molecular diagnostic for tuberculosis and rifampicin resistance. Future Microbiol, 6(9): 1067-82.

Lombardi, G., Di Gregori, V., Girometti, N., Tadolini, M., Bisognin, F., Dal Monte, P. 2017. Diagnosis of smear-negative tuberculosis is greatly improved by Xpert MTB/RIF. PLoS One, 12(4):e0176186. doi: 10.1371/j.pone.0176186.

Maynard-Smith, L., Larke, N., Peters, A.J. and Lawn, S.D., 2014. Diagnostic accuracy of the Xpert MTB/RIF assay for extrapulmonary and pulmonary tuberculosis when testing non-respiratory samples: a systematic Review. BMC Infectious Diseases, 14:709. DOI 10.1186/s12879- 


\section{4-0709-7.}

Moussa, S.M., Bayoumi, F.S. and Ali, A.M. 2016. Evaluation of GeneXpert MTB/RIF assay for direct diagnosis of pulmonary tuberculosis. Saudi Med J., 37(10): 10761081.

Nakwon, K., Choi, S.M., Lee, J., Park, Y.S., Chang-Hoon, L., Sang-Min, L., Chul-Gyu, Y., Kim, Y.W., Han, S.K. and Yim, J.J. 2013. Diagnostic accuracy and turnaround time of the Xpert MTB/RIF assay in routine clinical practice.PLos One, 8(10):e77456.

Pandey, P., Pant, N.D., Rijal, K.R., Shrestha, B., Kattel, S., Banjara, M.R., Maharjan, B. and Rajendra, K.C. 2017. Comparison between diagnostic accuracy of genotypic method using GeneXpert MTB/RIF assay and phenotypic methods using MGIT 960 conventional drug susceptibility testing method for the diagnosis of multidrugResistant Tuberculosis. PLOS, http://dx.doi.org/10.1371/journal.pone.0169 798.

Raj, A., Netrapal, S. and Promod, K. 2012. GeneXpert MTB/RIF Assay: a new hope for extrapulmonary tuberculosis. IOSR Journal of Pharmacy, 2 (1): 083-089.

Sajed, A.N., Khan, S., Butt, A.S., Srwar, A., Akhtar, R., Ahmad, I., Mukhtar, M.N., Sadaf, I., Akbar, H., Ali, S. andUsman, M. 2014. Rapid detection of Mycobacterium tuberculosis and Rifampicin Resistance in extra pulmonary samples using GeneXpert MTB/RIF assay. Journal of Dental and Medical Sciences, 13(11):50-53.

Shafiq, S., Saleem, F., Jabeen, K., Farooqi, J., Alam, W., Hanif, S., Ali, S., Shakoor, S., Hasan, R. 2016. Effect of time duration of digestion/ decontamination technique on yield of mycobacteria and contamination rates from sterile body fluids (pleural and ascitic fluid) and pus specimens. International Journal of Mycobacteriology. 5 Suppl 1: S195-S196. doi: 10.1016/j.ijmyco.2016.10.013.

Sharma, S.K., Mikashmi, K., Raj, N.Y., Jigyasa, C., Dinkar, B., Vishnubhatla, S., Rohini, S., and Binit, K. 2015. Evaluating the diagnostic accuracy ofXpert MTB/RIF assay in pulmonary tuberculosis. PLOS ONE DOI:10.1371/journal.pone.0141011.

Siddiqi, S.H., and Rusch Gerdes, S., 2006. MGIT procedure manual. Geneva, Switzerland: Foundation for Innovative New Diagnostics.

Singh, U.B., Urvashi, B.,Pooja, P., Mehta G., Anuj, K.B., Anant, M., Vinay, G., Vineet, A., Ranjani, R., Kuldeep, S.S., and Jyotish, C. 2016. Genotypic, phenotypic and clinical validation of GeneXpert in extra-Pulmonary and pulmonary TB in India. http://dx.doi.org/10.1371/journal.pone.0149 258.

Tang, T., Liu, F., Lu, X. and Huang, Q. 2017. Evaluation of GeneXpert MTB/RIF for detecting Mycobacterium tuberculosis in a hospital in China. Journal of International Medical Research, 45(2): 816-822.

Vadwai, V., Boehme, P.N., Shetty, A., Alland, D. and Rodrigues, C. 2011. Xpert MTB/RIF: a new pillar in diagnosis of extrapulmonary tuberculosis? J. Clin Microbiol, 49(7): 2540-5.

World Health Organization. 2016. Global tuberculosis report 2016.Geneva. www.who.int/TB.

\section{How to cite this article:}

Ehab Badran Rakha and Mohammed Abdel Razek Abdel Hakeem. 2017. GeneXpert MTB/RIF Assay: A Revolutionizing Method for Rapid Molecular Detection of Mycobacterium Tuberculosis in Comparison to Other Conventional Methods. Int.J.Curr.Microbiol.App.Sci. 6(10): 2573-2580. doi: https://doi.org/10.20546/ijcmas.2017.610.302 